\title{
Impact of metformin on disease control and survival in patients with head and neck cancer: a retrospective cohort study
}

\author{
Daniel J. Lee ${ }^{1 \dagger}$, Caitlin P. McMullen ${ }^{1 \dagger}$, Andrew Foreman ${ }^{1 \dagger}$, Shao Hui Huang ${ }^{2}$, Lin Lu ${ }^{3}$ Wei Xu ${ }^{3}$, John R. de Almeida',
} Geoffrey Liü ${ }^{3}$ Scott V. Bratman ${ }^{2}$ and David P. Goldstein ${ }^{1 *}$

\begin{abstract}
Objective: A number of in vitro and clinical studies have suggested potential antineoplastic effects of metformin. The impact of this medication on outcomes in head and neck cancer is less clear. Our aim was to determine the effect of metformin on outcomes within our large cohort of head and neck cancer patients with Type II Diabetes (T2DM).
\end{abstract}

Study design: Retrospective cohort study.

Setting: Tertiary Cancer Centre.

Subjects and methods: A retrospective review of individuals with head and neck squamous carcinoma (HNSCC) and T2DM between January 2005 and December 2011 at Princess Margaret Cancer Centre was conducted. Medication history was obtained from surveys at initial presentation and electronic medical record review. Using Cox regression analyses, the association between metformin use and local, regional and distant failures was explored. Subgroup analyses were conducted for oral cavity, oropharynx and larynx.

Results: A total of 329 HNSCC patients with T2DM were identified, including 195 metformin users and 134 nonmetformin users. Patients were well-matched in terms of clinical, pathologic, and treatment factors. No difference in local, regional, or distant failure was observed between diabetic metformin users and diabetic non-metformin users for the entire cohort or within subgroup analysis for subsite. No difference between the two groups was observed for overall survival, recurrence-free survival, and disease-specific survival at 5 years.

Conclusion: No association between metformin use and oncologic outcomes were observed in this large cohort of HNSCC patients. Multicenter, prospective studies may be needed to verify previous studies identifying a potential anti-neoplastic effect of this medication.

Keywords: Head and neck cancer, Diabetes, Metformin

\section{Introduction}

Metformin, an oral hypoglycemic agent, has been recognized for its potential antineoplastic effects. This low-cost, widely available, biguanide medication is indicated most commonly for the treatment of Type II diabetes mellitus

\footnotetext{
* Correspondence: david.goldstein@uhn.ca

${ }^{\dagger}$ Daniel J. Lee, Caitlin P. McMullen and Andrew Foreman contributed equally to this work

${ }^{1}$ Department of Otolaryngology Head and Neck Surgery/Surgical Oncology, University Health Network, Princess Margaret Cancer Center, University of Toronto, 610 University Ave 3-952, Toronto, ON M4V 2N8, Canada Full list of author information is available at the end of the article
}

(T2DM) and has a favorable adverse effect profile [1-3]. In vitro studies have demonstrated that metformin can potentiate the effects of chemotherapeutics and radiation as well as suppress tumor growth [4-8]. Clinically, metformin use in T2DM patients has been reported to be associated with improved oncologic outcomes in a number of cancers including breast, colorectal, prostate, thyroid, liver, and lung, compared to diabetic non-metformin users [9-14]. However, the results have been conflicting, with a recent meta-analysis of 11 randomized clinical trials involving 398 cancer types reporting no significant effect of metformin on all-cause mortality [14]. 
Table 1 Baseline characteristics of study population according to metformin use

\begin{tabular}{|c|c|c|c|c|}
\hline Co-variate & Full Sample $(n=329)$ & Non-Metformin $(n=134)$ & Metformin $(n=195)$ & $p$-value \\
\hline Primary Site (\%) & & & & 0.71 \\
\hline Oral cavity & $117(36)$ & $49(37)$ & $68(35)$ & \\
\hline Oropharynx & $80(24)$ & $36(27)$ & $44(23)$ & \\
\hline Hypopharynx & $13(4)$ & $5(4)$ & $8(4)$ & \\
\hline Larnyx & $119(36)$ & $44(33)$ & $75(38)$ & \\
\hline Age & & & & 0.9 \\
\hline Mean (sd) & $67.4(9.7)$ & $67.6(9.7)$ & $67.3(9.8)$ & \\
\hline Median (Min,Max) & $67.9(40.9,89.9)$ & $68.3(42.6,89.9)$ & $67.6(40.9,89.6)$ & \\
\hline Treatment Modality (\%) & & & & 0.67 \\
\hline Surgery & $73(22)$ & $30(22)$ & $43(22)$ & \\
\hline Adjuvant Rad & $38(12)$ & $14(10)$ & $24(12)$ & \\
\hline Adjuvant Chemo/Rad & $8(2)$ & $1(1)$ & $6(3)$ & \\
\hline Primary Rad & $159(48)$ & $66(49)$ & $93(48)$ & \\
\hline Primary Chemo/Rad & $52(16)$ & $23(17)$ & $29(15)$ & \\
\hline Extracapsular spread (\%) & & & & $0.04^{*}$ \\
\hline Absent & $52(84)$ & $24(96)$ & $28(76)$ & \\
\hline Present & $10(16)$ & $1(4)$ & $9(24)$ & \\
\hline Missing & 267 & 109 & 158 & \\
\hline Perineural invasion (\%) & & & & 0.49 \\
\hline No & $47(59)$ & $22(65)$ & $25(56)$ & \\
\hline Yes & $32(41)$ & $12(35)$ & $20(44)$ & \\
\hline Missing & 250 & 100 & 150 & \\
\hline CCI (\%) & & & & $<0.001^{*}$ \\
\hline 0 & $9(3)$ & $9(7)$ & $0(0)$ & \\
\hline 1 & $186(57)$ & $61(46)$ & $125(64)$ & \\
\hline 2 & $78(24)$ & $36(27)$ & $42(22)$ & \\
\hline $3+$ & $56(17)$ & $28(21)$ & $28(14)$ & \\
\hline Smoking (\%) & & & & 0.69 \\
\hline Never & $74(23)$ & $32(24)$ & $42(22)$ & \\
\hline Current/Ex & $252(77)$ & $101(76)$ & $151(78)$ & \\
\hline Missing & 3 & 1 & 2 & \\
\hline Drinking (\%) & & & & $0.029^{*}$ \\
\hline Never/Light & $188(63)$ & $67(55)$ & $121(68)$ & \\
\hline Moderate/Heavy/Ex & $111(37)$ & $54(45)$ & $57(32)$ & \\
\hline Missing & 30 & 13 & 17 & \\
\hline Clinical Stage (\%) & & & & 0.91 \\
\hline Early 0///II & $133(41)$ & $54(41)$ & $79(42)$ & \\
\hline Late III/IV & $190(59)$ & $79(59)$ & $111(58)$ & \\
\hline Missing & 6 & 1 & 5 & \\
\hline Stage cN (\%) & & & & 0.42 \\
\hline $\mathrm{NO} / \mathrm{Nx}$ & $190(59)$ & $77(58)$ & $113(59)$ & \\
\hline $\mathrm{N} 1$ & $23(7)$ & $7(5)$ & $16(8)$ & \\
\hline N2 & $100(31)$ & $46(35)$ & $54(28)$ & \\
\hline N3 & $11(3)$ & $3(2)$ & $8(4)$ & \\
\hline Missing & 5 & 1 & 4 & \\
\hline Stage cT (\%) & & & & 0.63 \\
\hline $\mathrm{T} 0 / \mathrm{Tx}$ & $1(0)$ & $0(0)$ & $1(1)$ & \\
\hline $\mathrm{T} 1$ & $81(25)$ & $28(22)$ & $53(28)$ & \\
\hline
\end{tabular}


Table 1 Baseline characteristics of study population according to metformin use (Continued)

\begin{tabular}{llll}
\hline Co-variate & Full Sample $(n=329)$ & Non-Metformin $(n=134)$ & Metformin $(n=195)$ \\
\hline T2 & $101(32)$ & $45(35)$ & $56(29)$ \\
T3 & $77(24)$ & $33(25)$ & $44(23)$ \\
T4a/4b & $60(19)$ & $24(18)$ & $36(19)$ \\
Missing & 9 & 4 & 5 \\
Staging cM (\%) & & & \\
M0 & $327(99)$ & $134(100)$ & $193(99)$ \\
M1 & $2(1)$ & $0(0)$ & $2(1)$ \\
Follow-up time & & & $3.1(2.1)$ \\
Mean (sd) & $3.1(2.1)$ & $3(2.2)$ & $2.8(0,8.4)$ \\
Median (Min, Max) & $2.7(0,8.8)$ & $2.7(0,8.8)$ & 0.69 \\
\hline
\end{tabular}

* indicates statistical significance

$\mathbf{a}$
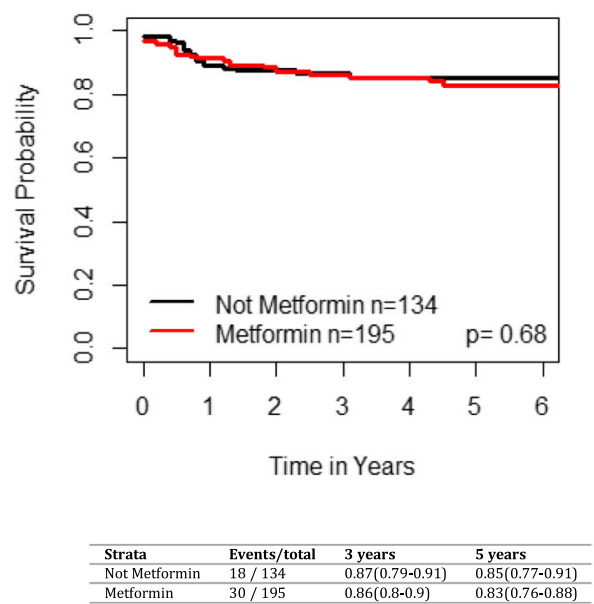

\begin{tabular}{llll}
\hline Metformin & $30 / 195$ & $0.86(0.8-0.9)$ & $0.83(0.76-0.88)$ \\
\hline Total & $48 / 329$ & $0.86(0.82-0.9)$ & $0.84(0.79-0.88)$ \\
\hline
\end{tabular}

C
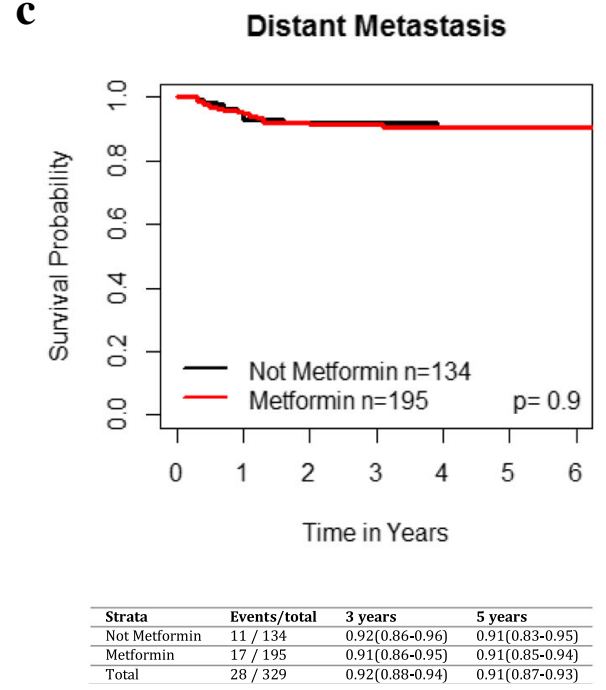

Fig. 1 Impact of metformin use in T2DM across all subsites on a) local failure, b) regional failure, and c) distant failure. Kaplan-Meier analysis with associated $p$ values for the entire grouping is demonstrated b Regional control
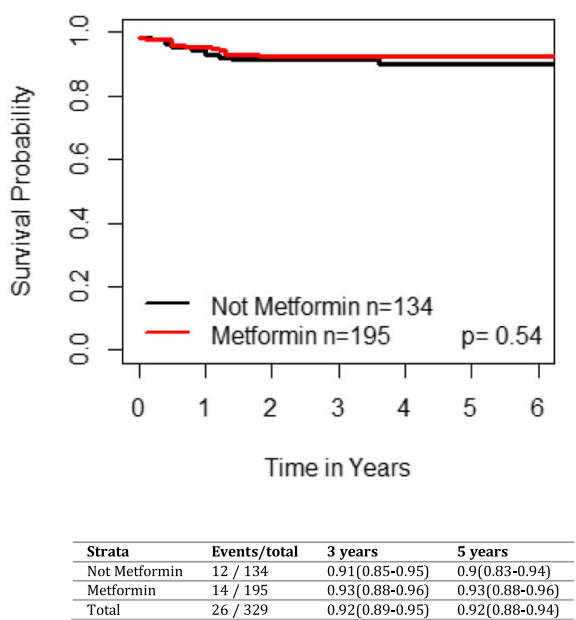

\begin{tabular}{llll} 
Metformin & $14 / 195$ & $0.93(0.88-0.96)$ & $0.93(0.88-0.96)$ \\
\hline Total & $26 / 329$ & $0.92(0.89-0.95)$ & $0.92(0.88-0.94)$ \\
\hline
\end{tabular} 
$\mathbf{a}$
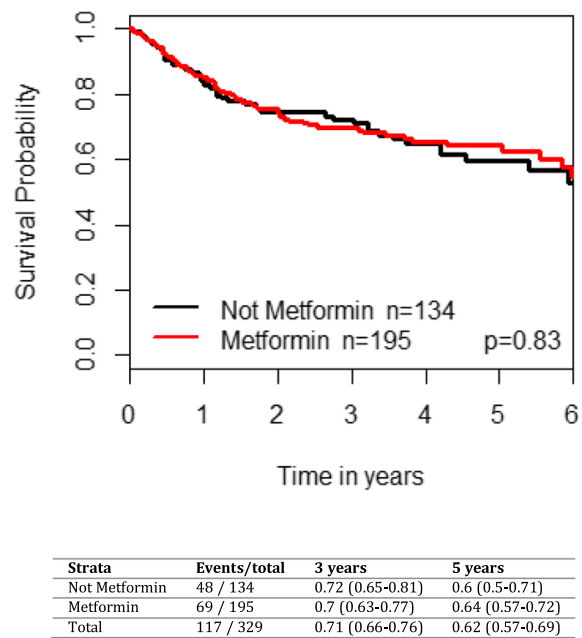

\begin{tabular}{llll} 
Mettormin & $69 / 195$ & $0.7(0.63-0.77)$ & $0.64(0.07-0.72)$ \\
\hline Total & $117 / 329$ & $0.71(0.66-0.76)$ & $0.62(0.57-0.69)$ \\
\hline
\end{tabular}

c

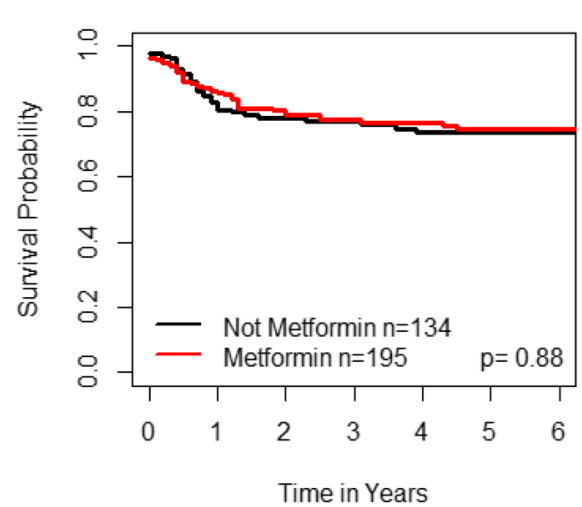

b

Disease specific survival

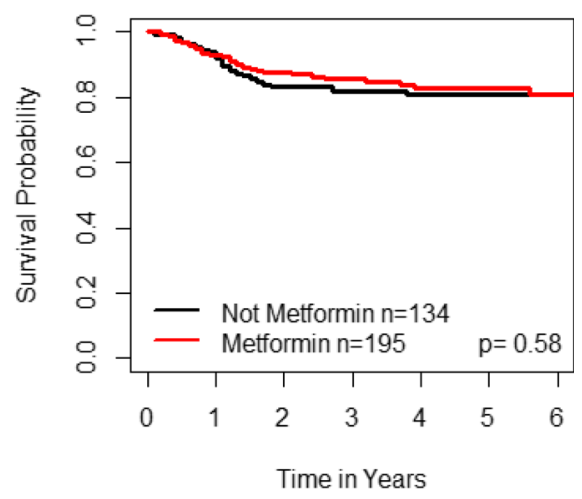

\begin{tabular}{llll}
\hline Strata & Events/total & $\mathbf{3}$ years & $\mathbf{5}$ years \\
\hline Not Metformin & $24 / 134$ & $0.82(0.74-0.88)$ & $0.81(0.72-0.87)$ \\
\hline Metformin & $31 / 195$ & $0.86(0.8-0.9)$ & $0.83(0.76-0.88)$ \\
\hline Total & $55 / 329$ & $0.84(0.8-0.88)$ & $0.82(0.77-0.86)$ \\
\hline
\end{tabular}

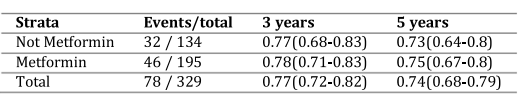

S

Fig. 2 Impact of metformin use in T2DM patients across all subsites on a) overall survival, b) disease specific survival and c) recurrence-free survival. Kaplan-Meier analysis with associated $p$ values is demonstrated

There have been a few studies assessing the impact of metformin use on outcomes in patients with head and neck squamous cell carcinoma (HNSCC) with mixed results $[15,16]$. We have previously reported that the presence of T2DM alone does not adversely effect cancer survival outcomes among HNSCC patients managed at the Princess Margaret Cancer Center despite the presumed burden of diabetic comorbidity [17]. The objective of this study was to determine if metformin use in T2DM patients was independently associated with improved oncologic outcomes across the different subsites in HNSCC.

\section{Methods}

\section{Study cohort and data acquisition}

Following approval by the institutional Research Ethics Board, a retrospective chart review was performed of patients with a newly diagnosed, previously untreated, squamous cell carcinoma of the oral cavity, oropharynx, larynx and hypopharynx managed in our institution between January 2005 and December 2011. Patients were eligible for inclusion if they were $>18$ years of age, had a diagnosis of T2DM and received curative intent treatment. Patients were identified through the institutional Cancer Registry and from a radiation oncology prospective anthology of outcomes database (Anthology Database) of all radiation oncology patients in our institution [18].

Sociodemographic information, treatment details, pathologic data and outcomes were obtained from the Anthology Database and supplemented by chart review. The T2DM patients identified in our previous study on outcomes of patients with HNSCC with and without T2DM were included in the current study [17]. Comorbidities and medication history (primarily metformin use and combination oral medications that contained 
$\mathbf{a}$

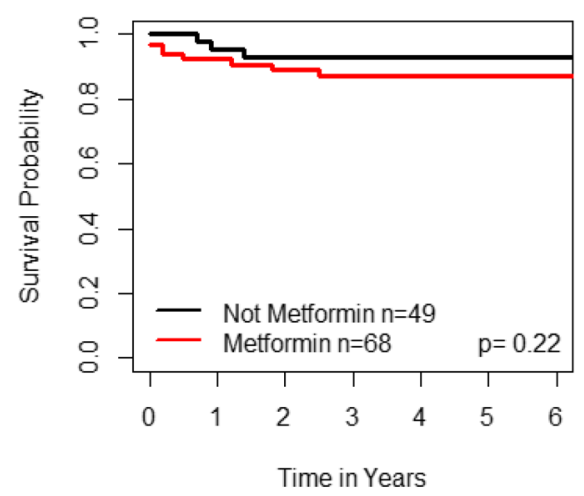

\begin{tabular}{llll} 
Strata & Events/total & 3 years & 5 years \\
\hline Not Metformin & $3 / 49$ & $0.93(0.78-0.98)$ & $0.93(0.78-0.98)$ \\
\hline
\end{tabular} \begin{tabular}{llll} 
Not Metformin & $3 / 49$ & $0.93(0.78-0.98)$ & $0.93(0.78-0.98)$ \\
\hline Metformin & $9 / 68$ & $0.87(0.75-0.93)$ & $0.87(0.75-0.93)$ \\
\hline
\end{tabular}

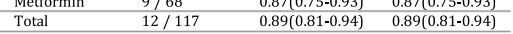

c

Distant Metastasis

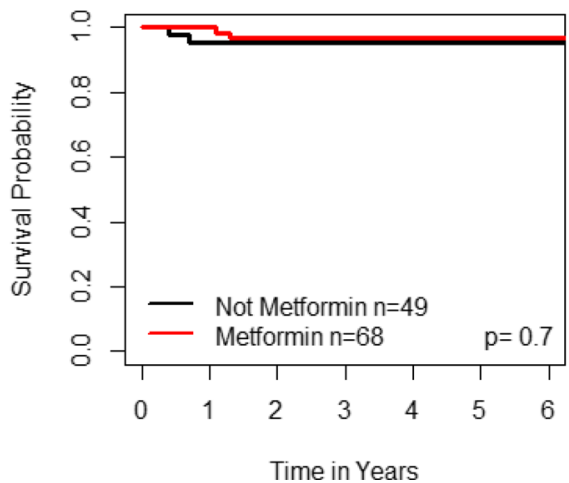

b
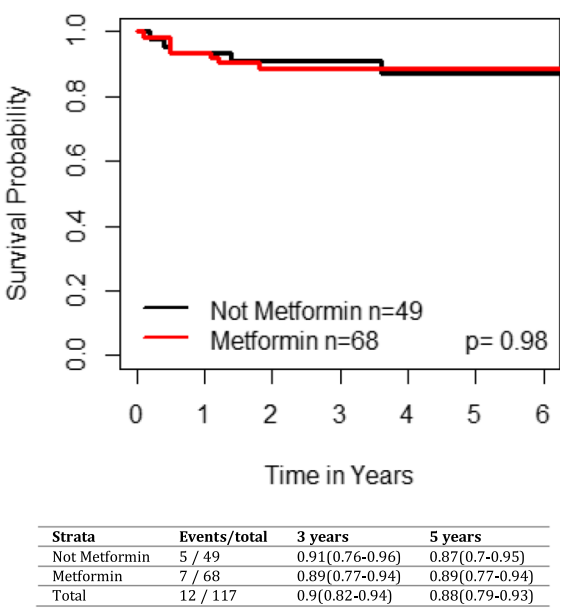

Total

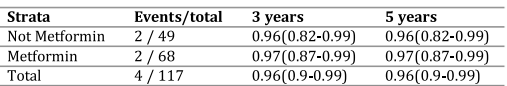

$4 / 117$ $0.96(0.9-0.99)-0.9$

Fig. 3 Impact of metformin use in T2DM patients with oral cavity cancer on a) local failure, b) regional failure, and c) distant failure. Kaplan-Meier analysis with associated $p$ values is demonstrated

metformin) were obtained from chart review which were then cross-referenced with two additional databases, if available: (1) The Clinical Ambulatory Information System (CAIS) electronic preoperative anesthesia record for patients managed with primary surgery and (2) the head and neck translational research database, which has aimed to prospectively enroll all patients with a new diagnosis of HNSCC at Princess Margaret Cancer Center. The latter database requires patients complete a detailed medical and medication history, including selfreported diabetes. All patients were staged using the 7th edition of the AJCC/UICC Cancer Staging Manual [19]. Treatment details at our center have been described in the previous publication [17].

\section{Statistical analysis}

Patients using metformin at the time of their HNSCC diagnosis were classified as Type 2 Diabetic Metformin positive $(\mathrm{T} 2 \mathrm{DMM}+)$. Summary statistics were provided for clinical and demographic variables. Baseline patient characteristics were compared between metformin and non-metformin users. In an attempt to better delineate the effects of metformin, we focused on the T2DM group only, which is also in keeping with other head and neck studies $[15,16]$. Differences between the two groups were examined by chi-square $\left(x^{2}\right)$ test and T test for categorical variables and continuous variables, respectively. The clinical end points were control rates and survival. Overall survival (OS) was defined as time from date of diagnosis to death of any cause, sensored at last last follow-up date; recurrence-free survival was defined as time from date of diagnosis to any failure, sensored at the last follow-up date. Death without failure is considered as competing risk. The Kaplan-Meier method was used to estimate the probabilities for OS, RFS, DSS, local control (LC), regional control (RC) and distant 
$\mathbf{a}$
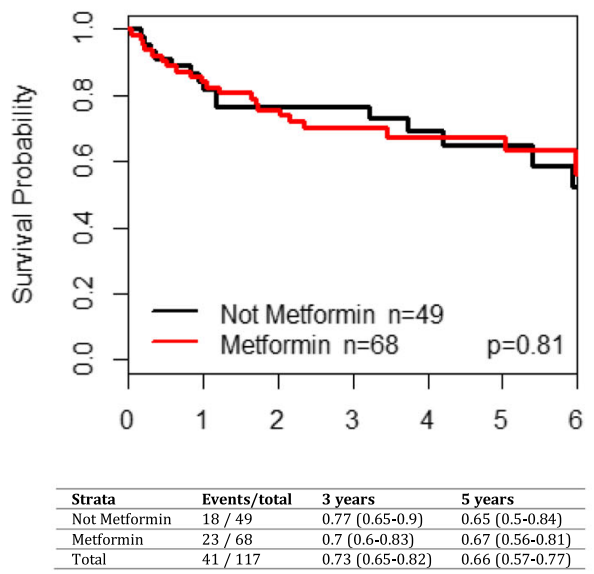

\begin{tabular}{llll} 
Metformin & $23 / 68$ & $0.7(0.6-0.83)$ & $0.67(0.56-0.81)$ \\
\hline Total & $41 / 117$ & $0.73(0.65-0.82)$ & $0.66(0.57-0.77)$ \\
\hline
\end{tabular}

c

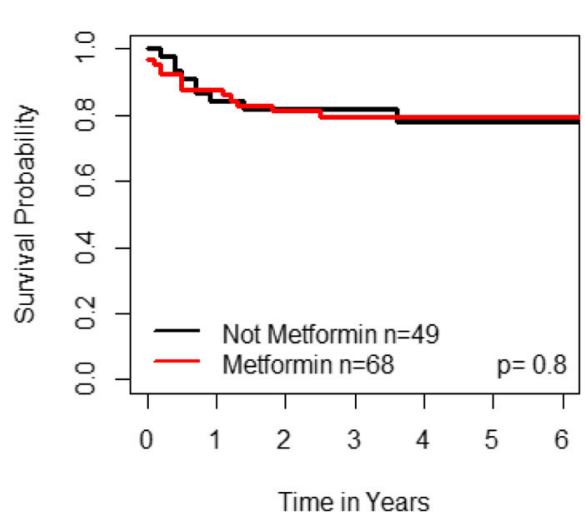

b

Disease specific survival
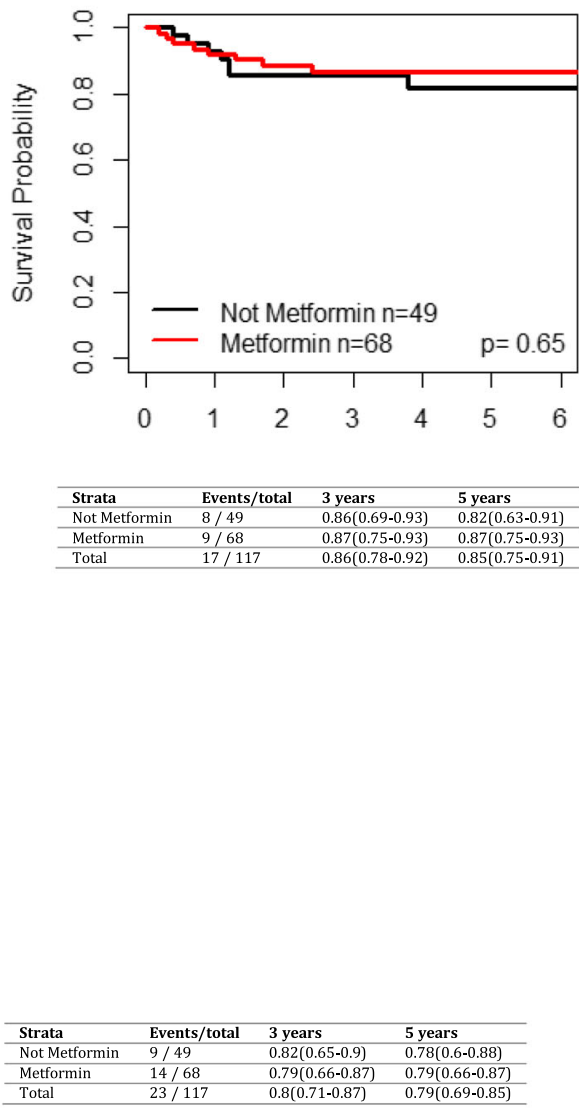

\begin{tabular}{llll}
\hline Metformin & $14 / 68$ & $0.79(0.66-0.87)$ & $0.79(0.66-0.87)$ \\
\hline Total & $23 / 117$ & $0.8(0.71-0.87)$ & $0.79(0.69-0.85)$ \\
\hline
\end{tabular}

Fig. 4 Impact of metformin use in T2DM patients with oral cavity cancer on a) overall survival, b) disease specific survival and c) recurrence-free survival. Kaplan-Meier analysis with associated $p$ values is demonstrated

control (DC) for the entire cohort and then, in an exploratory manner, for the individual head and neck subsites. Analyses were performed for individual subsites to minimize potential difference in clinical behavior atributable to variation in anatomy and tumor biology. The analyzed subsites included oral cavity (OC), oropharynx, and larynx. Hypopharynx was excluded from specific subsite analysis due to very small numbers in our cohort. Survival and control rates between metformin users with non-metformin users were compared by log-rank test. A two-sided test was applied with alpha set at 0.05 for statistical significance. Cox proportional hazards regression models were used for univariable (UVA) and and multivariable analysis (MVA). Variables included for MVA were clinical stage and comorbidity due to their clinical importance. Hazard ratios and corresponding 95\% confidence intervals were provided. The proportionality assumption was tested using residuals. All statistical analyses were conducted using SAS 9.3 and R.

\section{Results}

\section{Study population}

There were 329 patients with T2DM eligible for inclusion, of which 195 (59\%) were taking metformin at the time of presentation. The median age of the cohort was 67.9 years. Larynx was the most common cancer subsite (36\%), followed by oral cavity (36\%), oropharynx (24\%), and hypopharynx (4\%). Table 1 summarizes the baseline characteristics of the patient cohort. Statistically significant differences between the T2DMM+ group and T2DMM- groups included alcohol consumption history, with heavier drinkers in the non-metformin cohort ( $p=$ 


\section{a}
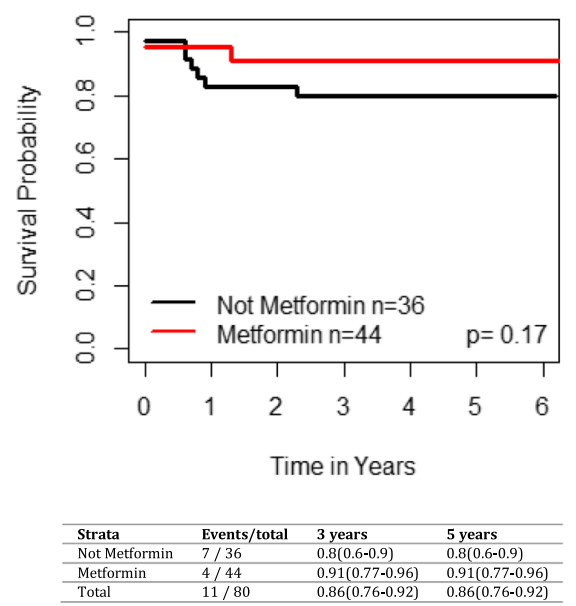

c

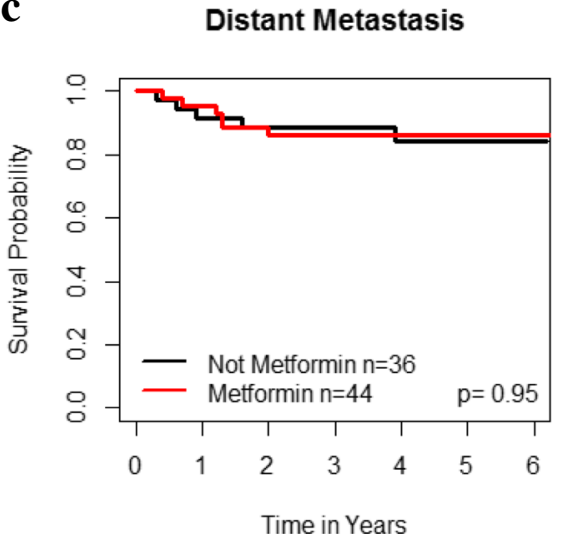

b
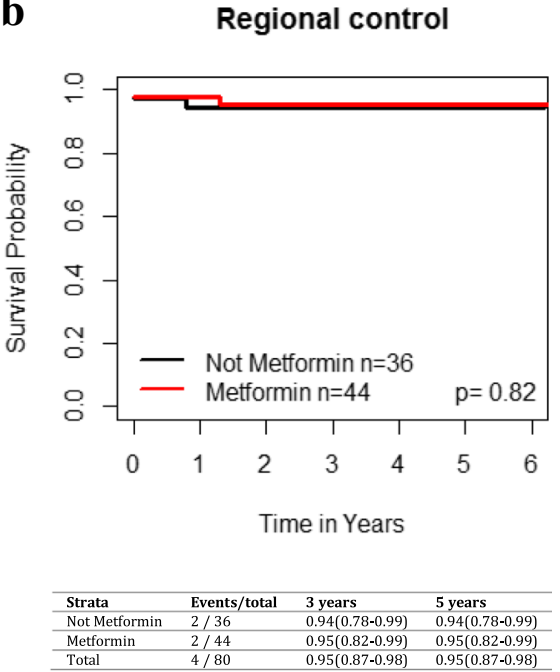

\begin{tabular}{llll} 
Metformin & $2 / 44$ & $0.95(0.82-0.99)$ & $0.95(0.82-0.99)$ \\
\hline Total & $4 / 80$ & $0.95(0.87-0.98)$ & $0.95(0.87-0.98)$
\end{tabular}

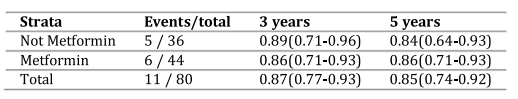

\begin{tabular}{llll}
\hline Metformin & $5 / 44$ & $0.86(0.71-0.93)$ & $0.86(0.71-0.93)$ \\
\hline Total & $11 / 80$ & $0.87(0.77-0.93)$ & $0.85(0.74-0.92)$ \\
\hline
\end{tabular}

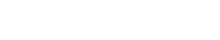

Fig. 5 Impact of metformin use in T2DM patients with oropharynx cancer on a) local failure, b) regional failure, and c) distant failure. KaplanMeier analysis with associated $p$ values is demonstrated

0.029); greater pathologic extracapsular spread (ECS) was observed in the metformin cohort $(p=0.04)$ and higher Charlson Comorbidity Index (CCI) score in the non-metformin cohort $(p=<0.001)$. The mean and median follow up times for the entire cohort were 3.1 and 2.7 years, respectively.

\section{All HNSCC patients with T2DM}

On UVA, metformin use was not significantly associated with improved LC (HR 1.13 [95\% CI 0.63-2.02], $p=$ 0.68), regional control (HR 0.78 [95\% CI 0.36-1.69], $p=$ 0.54) or distant control (HR 1.05 [95\% CI 0.49, 2.24], $p=0.9$ ) (Fig. 1). For the entire cohort, the 3 and 5 year OS was $71 \%$ [ $95 \%$ CI $66-76 \%$ ] and $62 \%$ [95\% CI $57-$ $69 \%$ ], respectively. The 3 and 5 year OS, RFS and DSS for the overall cohort based on metformin use are shown in Fig. 2. There was no significant difference in overall survival $\quad\left(\mathrm{HR} \quad 1.04 \quad\left[\begin{array}{llll}95 \% & \mathrm{CI} & 0.72-1.5\end{array}\right], \quad p=0.83\right)$, recurrence-free survival (HR 1.04 [95\% CI 0.66-1.62], $p=0.88$ ) and disease-specific survival (HR 1.16 [95\% CI $0.68-1.98], p=0.58$ ) between metformin and nonmetformin users.

\section{Exploratory analysis of T2DM patients with Oral cavity cancers}

In the OC subgroup, there were 49 (42\%) nonmetformin users and 68 (58\%) metformin users. On UVA, metformin use was not significantly associated with improved LC (HR 0.46 [95\% CI 0.12-1.76], $p=$ 0.24), regional control (HR 1.02 [95\% CI 0.33-3.15], $p=$ 0.98 ) or distant control ( $p=0.69$, HR 1.47 (95\%CI $0.22-$ 9.85) during the follow up period (Fig. 3). There were no significant differences in OS $(p=0.8$, HR 1.08 [95\% CI $0.58-2.01])$, RFS ( $p=0.8$, HR 0.9 [95\% CI $0.39-2.05]$ ) or DSS $(p=0.63$, HR 1.26 [95\% CI 0.49-3.22]) between T2DMM+ and T2DMM- groups (Fig. 4). 
a

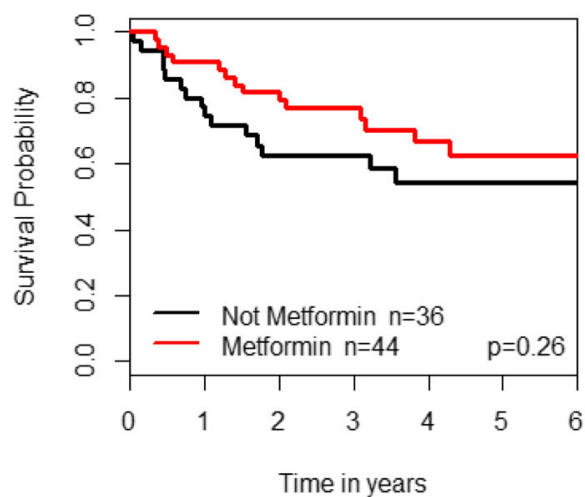

\begin{tabular}{llll} 
Strata & Events/total & $\mathbf{3}$ years & $\mathbf{5}$ years \\
\hline
\end{tabular} \begin{tabular}{llll} 
Not Metformin & $15 / 36$ & $0.63(0.48-0.81)$ & $0.54(0.39-0.75)$ \\
\hline Metformin & $14 / 44$ & $0.77(0.65-0.91)$ & $0.62(0.48-0.81)$ \\
\hline
\end{tabular}

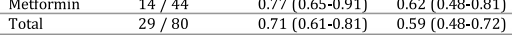

c Recurrence-free Survival

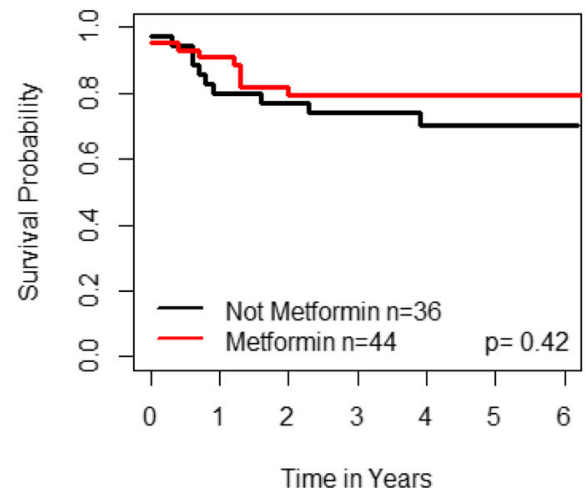

Time in Years b

Disease specific survival

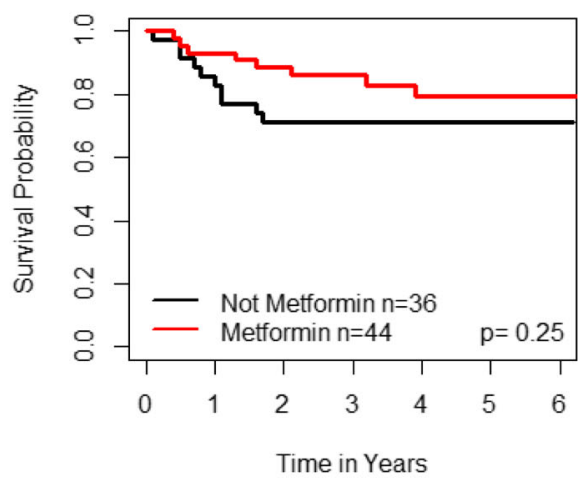

\begin{tabular}{llll} 
Strata & Events/total & 3 years & 5 years \\
\hline Not Metformin & $10 / 36$ & $0.71(0.51-0.83)$ & $0.71(0.51-0.83)$ \\
\hline
\end{tabular} \begin{tabular}{llll} 
Not Metformin & $10 / 36$ & $0.71(0.51-0.83)$ & $0.71(0.51-0.83)$ \\
\hline Metformin & $8 / 44$ & $0.86(0.71-0.93)$ & $0.79(0.6-0.89)$ \\
Total & $18 / 80$ & $0.8(0.68-0.87)$ & $0.76(0.63-0.84)$
\end{tabular}

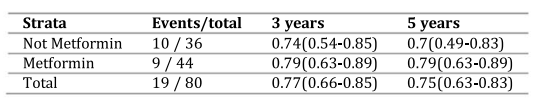

Fig. 6 Impact of metformin use in T2DM patients with oropharynx cancer on a) overall survival, b) disease specific survival and c) recurrence-free survival. Kaplan-Meier analysis with associated $p$ values is demonstrated

Exploratory analysis of T2DM patients with oropharynx There were 44 oropharynx cancer (OPC) patients on metformin and 36 diabetic OPC patients not taking metformin. Metformin use in T2DM OPC patients was not significantly associated with improved LC (HR 2.31 [95\% CI $0.68-7.79], p=0.18$ ), regional control (HR 1.25 [95\% CI 0.18-8.68], $p=0.82$ ) or distant control (HR 1.04 [95\% CI 0.32-3.34], $p=0.95$ ) (Fig. 5). The 3 and 5 year OS, RFS, and DSS for OP patients are shown in Fig. 6. There were no significant differences in OS ( $p=0.26$, HR 1.52 [95\% CI $0.73-3.16])$, RFS $(p=0.42$, HR 1.44 [95\% CI 0.59-3.51]), and DSS $(p=0.25$ HR 1.72 [95\% CI 0.69-4.32]) between the two cohorts of OPC patients on UVA. Given the differences in biology of HPV-related and HPVunrelated OPC, we performed an additional exploratory analysis of outcomes in metformin and non- metformin group based on p16 status, acknowledging that the results need to be interpreted with caution given the limited numbers. There were a total of 67 OPC patients with known HPV status, including 47 p16+ patients (70\%) and 20 p16 negative patients (30\%). Within the p16+ cohort of T2DM OPC patients, there were no differences between the metformin and non-metformin patients in terms of OS ( $p=$ $0.59)$, RFS $(p=0.71)$, DSS $(p=0.62)$, local control $(p=$ $0.74)$, regional control $(p=0.43)$ and distant control $(p=0.76)$. For $\mathrm{p} 16$ negative OPC diabetic patients, similarly, there were no differences between metformin and non-metformin groups in terms of OS ( $p=$ $0.14)$, RFS $(p=0.56)$, DSS $(p=0.32)$, local control $(\mathrm{p}=$ $0.56)$, regional control $(p=0.5)$, and distant control $(p=0.29)$. Of note, there were very few local and regional failures in this population. 


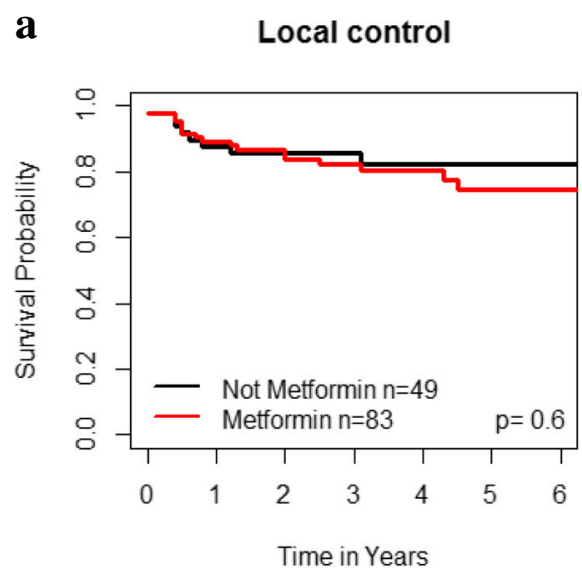

b
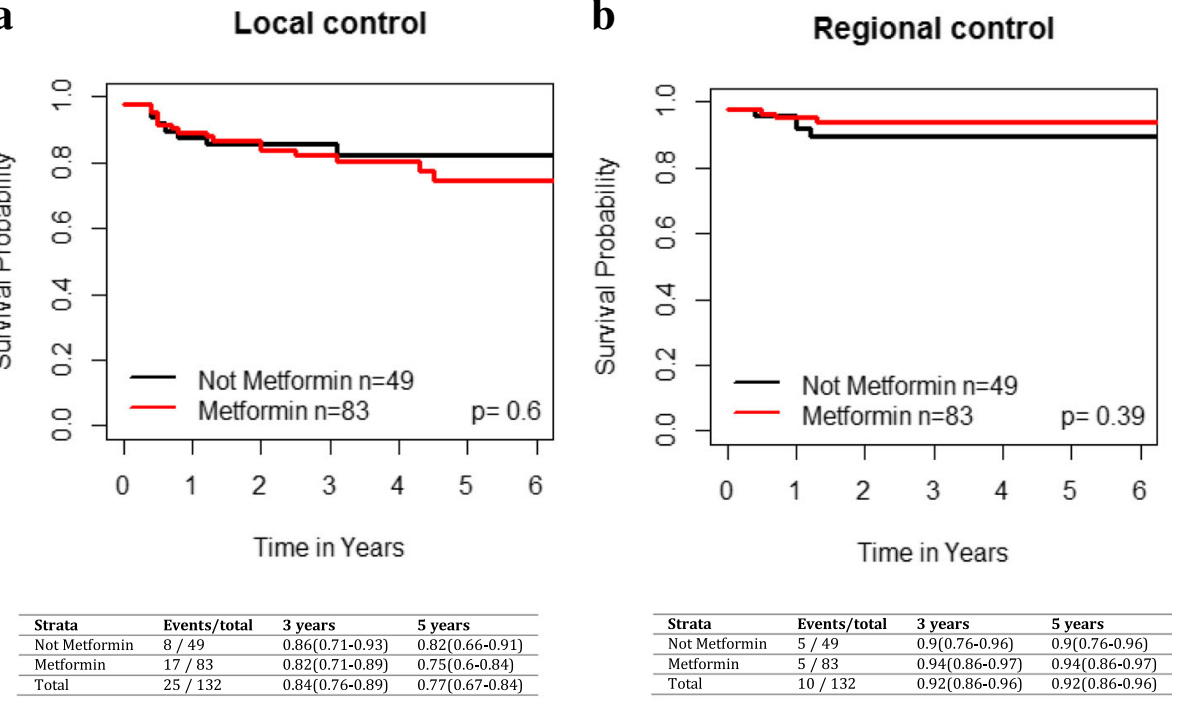

c

Distant Metastasis
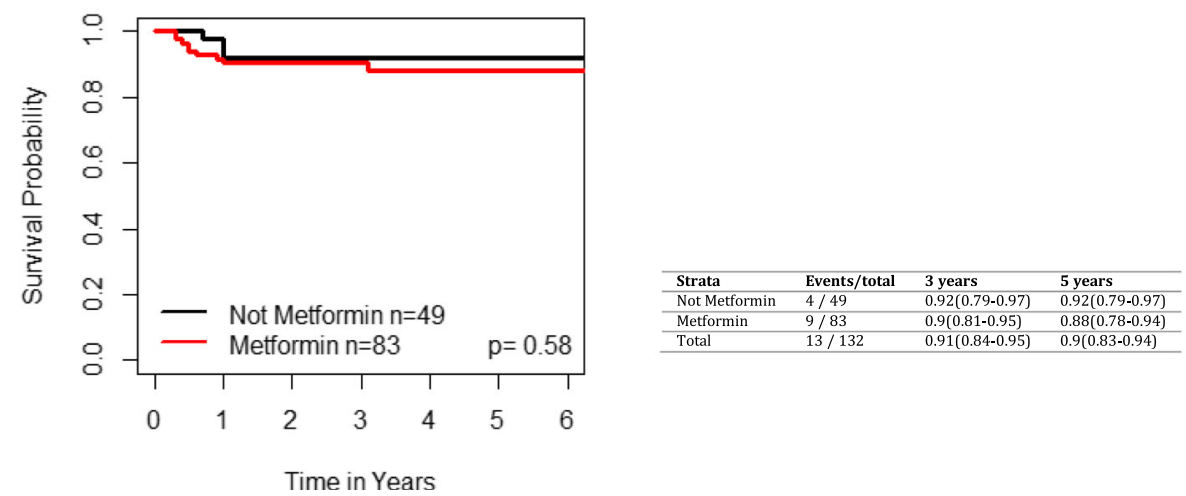

Fig. 7 Impact of metformin use in T2DM patients with larynx cancer on a) local failure, b) regional failure, and c) distant failure. Kaplan-Meier analysis with associated $p$ values is demonstrated

Exploratory analysis of T2DM patients with larynx Cancer There were 75 larynx cancer patients with T2DM on metformin and 44 T2DM patients not on metformin. The 3 and 5 year local, regional, and distant control rates for the T2DM laryngeal cancer patients based on metformin use are presented in Fig. 7. Metformin use was not significantly associated with improved LC $(p=0.87, \mathrm{HR} 0.93$ [95\% CI 0.4-2.19]), regional control $(p=0.24$, HR 2.17 (95\% CI $0.59-8)$ ) or distant control $(p=0.78$, HR 0.83 [95\% CI 0.21-3.22]). There were no significant differences in OS $(p=0.28$, HR 0.69 [95\% CI 0.35-1.36]), RFS ( $p=$ 0.75, HR 1.12 [95\% CI 0.55-2.3]), and DSS ( $p=0.73 \mathrm{HR}$ 0.83 [95\% CI 0.29-2.38]) between the two cohorts of laryngeal cancer patients upon univariate analysis (Fig. 8).

\section{Discussion}

Metformin was originally noted to have potential oncologic benefits in an observational study published in
2005, which reported that its use was associated with a decreased incidence of cancer [20]. One of the proposed mechanisms for its anti-neoplastic effects is the reduction of systemic levels of insulin and insulin-like growth factor-1 (IGF-1), thus lowering the mitogenic activity of hyperinsulinemia [21-23]. In addition, it is hypothesized that metformin inhibits the mTOR pathway and activation of AMP-activated protein kinase (AMPK), leading to the blockade of cell proliferation in cancer cells [2325]. Dysregulation of mTOR signaling has been identified as a targetable oncogenic pathway in HNSCC [24]. In certain breast cancers, the cell adhesion protein CD24+ plays a role in the development of distant metastases and is suppressed by metformin in vitro [26]. CD24+ has also been associated with aggressive features in HNSCC cell lines [27]. Studies included in a recent systematic review of the anti-tumor effects on head and neck cancer cell lines reported that metformin plays a 
$\mathbf{a}$
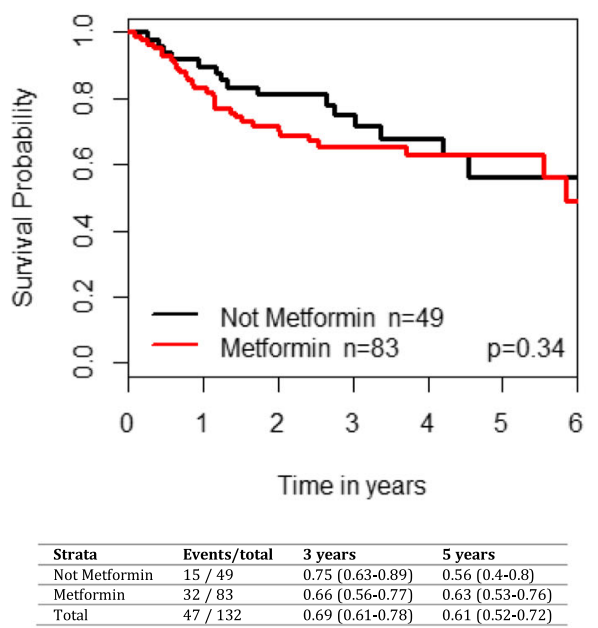
Metforn

c

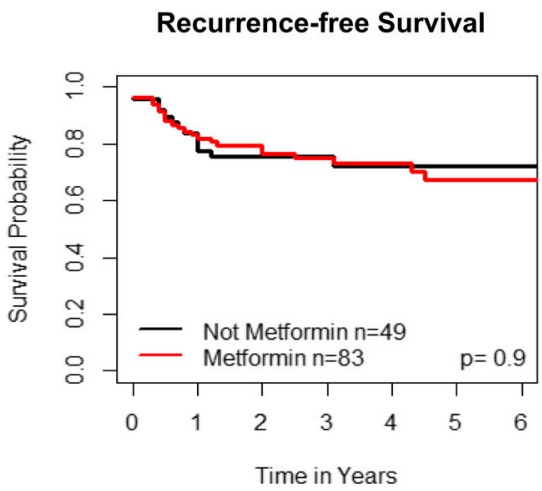

b
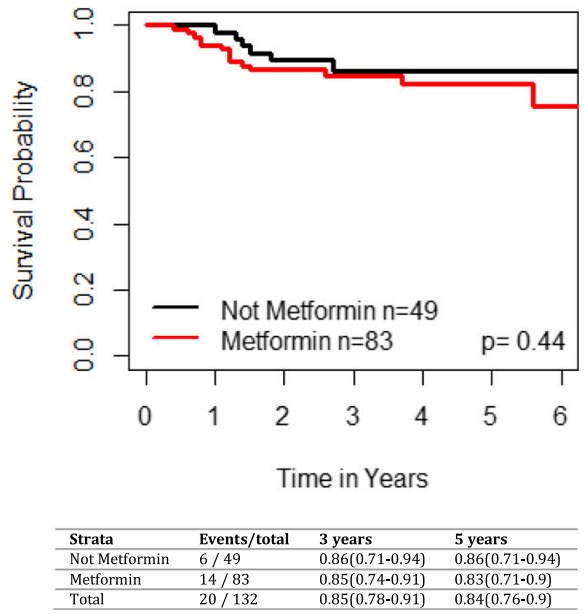

\begin{tabular}{llll} 
Metformin & $14 / 83$ & $0.85(0.74-0.91)$ & $0.83(0.71-0.9)$ \\
\hline Total & $20 / 132$ & $0.85(0.78-0.91)$ & $0.84(0.76-0.9)$
\end{tabular}

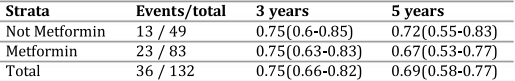

Fig. 8 Impact of metformin use in T2DM patients with larynx/hypopharynx cancer on a) overall survival, b) disease specific survival and c) recurrence-free survival. Kaplan-Meier analysis with associated $p$ values is demonstrad

role in proliferation inhibition, apoptosis, G0/G1 cell cycle arrest, and carcinogenic pathway protein regulation [28].

Given that metformin is a low cost, well-tolerated medication that has minimal toxicity, it would be an ideal anti-cancer agent. While some clinical studies have demonstrated that the use of metformin results in potentially increased overall survival and decreased incidence of cancer in multiple sites and cell types, such as colon, breast and liver $[9,10,13,29-31]$, there are contradicting randomized controlled trials and observational studies that have failed to demonstrate an unequivocally beneficial effect of metformin use on cancer survival [14, 32-34]. In HNSCC, the data are limited and results of existing studies are similarly mixed, particularly with respect to reporting the effects of metformin on outcomes such as survival or recurrence. One of the earliest observational reports in the head and neck cancer literature was by Skinner et al. who found that patients with oral cavity, oropharynx (HPV status not reported), larynx and hypopharynx cancer on metformin at the time of radiation had a lower locoregional recurrence rate compared to controls matched for tumor and nodal stage, surgical margin status, and TP53 status $(p=0.04)$ [7]. However, the results need to be interpreted with caution with only 10 patients in the metformin group. Sandulache et al. examined the observational association between metformin use and outcomes in glottic and supraglottic laryngeal carcinoma [15]. They included 21 diabetic patients on metformin and 22 diabetic patients not taking metformin. In these relatively small groups, metformin use was marginally significant but of a great magnitude of benefit in improving overall survival (OS) (OR, 3.0; 95\% $\mathrm{CI}, 1.0-8.4 ; p=.04)$; of note, there was no difference in RFS between the two groups. Given the limited number of patients and outcomes (i.e. death) the multivariable 
analysis must be interpreted with caution. It could not be excluded that the deaths were actually due to noncancer reasons (e.g. cardiovascular disease) that could be linked to metformin use (e.g., use of metformin may represent better T2DM care). Patients seeking care for their diabetes and taking metformin may seek out other healthy behaviors to modify their risk, potentially resulting in skewed survival results [35]. In addition, comorbidities were not assessed. While HbA1c levels were similar between groups, it is difficult to ascertain whether the improved survival is purely attributable to the effect of metformin without accounting for the comorbidities. The primary endpoints of their study were OS and RFS; information specifically regarding distant and locoregional control were not reported. Similar to our results, Yen et al. using a sample of diabetic patients enrolled in the National Health Insurance Program of Taiwan reported that while metformin use was associated with a reduction in the incidence of developing head and neck cancers, there was no significant difference in overall survival between patients with diabetes in the metformin positive $(n=195)$ and metformin negative $(n=290)$ cohorts who subsequently developed head and neck cancer (sites included oral cavity, oropharynx, larynx, hypopharynx, salivary gland, nasopharynx and nasal/sinus) ${ }^{16}$. In the most recent study, Spratt et al. performed a retrospective review of 184 diabetic OPC patients treated with primary radiation, of which 102 were on metformin [36]. They reported improved distant metastasis-free survival for the cohort of patients on metformin; however, there was no difference observed for 5-year local and regional failure-free survival. There was no difference between metformin and nonmetformin in terms of proportion of patients that were HPV positive. Our study failed to demonstrate a benefit of metformin use in OPC patients, for either the HPV positive and negative groups. Similarly, across oral cavity and larynx cancer patients there was no benefit of improved survival with metformin use amongst diabetic patients.

In terms of comparing clinical results with preclinical studies, the latter utilized much higher doses of metformin than the conventional dose used in the treatment of diabetes $[8,37,38]$. Considering the pharmacokinetics, the actual quantity of metformin delivered to cancer cells may be insufficient to truly demonstrate its anti-proliferative effects $[39,40]$. These shortcomings of the studies render the results less generalizable to human subjects who are taking metformin at a much lower dose. There are some limitations of the current study that needs to be considered. Patients included in this study were defined as metformin-users based on use at the time of diagnosis, but the duration of use, dosage, medication compliance, and medication changes were not incorporated into analysis, as this information was not available from retrospective chart review. As reported by Suissa et al., this may lead to time-related biases [33]. For example, Suissa et al. describe immortal time bias which occurs when the time prior to the medication exposure is misclassified and may skew results towards risk reduction [33]. Timerelated bias issues are not addressed by many authors reporting differential outcomes between metformin users and diabetic non-metformin users. Confounding factors including renal and hepatic function affecting drug metabolism and glucose control were also not included. Furthermore, the prescription of metformin over injectable insulin or other oral hypoglycemic drugs may indicate diabetic severity, which is known to affect survival outcomes. Lastly, while the study is one of the largest institutional series, it is limited by the number of diabetic patients as well as the number of patients lost to follow up. Given the small sample size in the subset analyses the results have to be interpreted with caution and while metformin was not associated with recurrence or survival in our cohort this may be due lack of power to demonstrate such a finding. However, other studies mentioned in the discussion that reported an effect of metformin on outcomes also had relatively small sample size. In addition, the metforming usage was obtained via retrospective review, and we are unable to explore the potential influence of the duration, dosage and the intensity of metformin on outcome of head and neck cancer patients.

The impact of a new agent with a favorable side effect profile for effective adjuvant therapy in HNSCC would be enormous. However, the clinical data thus far has failed to definitively demonstrate a significant effect on outcomes in metformin users. This study reinforces prior work that suggest this drug may not be useful in HNSCC patients overall or by subsite.

\section{Conclusion}

In our HNSCC cohort, the use of metformin was not associated with improved overall survival, disease-specific survival, local, regional, or distant control overall or within any subsite. Additional prospective multi-centre studies are needed to further elucidate the role of metformin in this disease.

\footnotetext{
Authors' contributions

Study concept and design: DJL, AF, DPG, Acquisition of data: DJL, AF, SHH, Analysis and interpretation of data: DJL, CPM, DPG, Critical revision of the manuscript ofr important intellectual content: DJ, CPM, AF, SHH, JDA, LL, SVB, DPG, Statistical analysis: LL, WX, Administrative, technical or material support: DJL, DPG, Study supervision: Goldstein. All authors read and approved the final manuscript.
} 


\section{Availability of data and materials}

Dr. David P. Goldstein and Dr. Daniel J. Lee had full access to the study and take full responsibility for the integrity and accuracy of data analysis.

\section{Ethics approval and consent to participate}

Institutional REB approval from the University Health Network.

\section{Consent for publication}

Not applicable.

\section{Competing interests}

The authors declare that they have no competing interests.

\section{Author details \\ 'Department of Otolaryngology Head and Neck Surgery/Surgical Oncology, University Health Network, Princess Margaret Cancer Center, University of Toronto, 610 University Ave 3-952, Toronto, ON M4V 2N8, Canada. ${ }^{2}$ Department of Radiation Oncology, University Health Network, Princess Margaret Cancer Centre, University of Toronto, Toronto, ON, Canada. ${ }^{3}$ Biostatistics Department, Princess Margaret Cancer Centre and Dalla Lana School of Public Health, University of Toronto, Toronto, ON, Canada.}

Received: 1 August 2018 Accepted: 21 May 2019

Published online: 25 July 2019

\section{References}

1. Scarpello JH, Howlett HC. Metformin therapy and clinical uses. Diab Vasc Dis Res. 2008;5(3):157-67.

2. Knowler WC, Barrett-Connor E, Fowler SE, Hamman RF, Lachin JM, Walker EA, et al. Reduction in the incidence of type 2 diabetes with lifestyle intervention or metformin. N Engl J Med. 2002;346(6):393-403.

3. Jakubowicz DJ, luorno MJ, Jakubowicz S, Roberts KA, Nestler JE. Effects of metformin on early pregnancy loss in the polycystic ovary syndrome. J Clin Endocrinol Metab. 2002:87(2):524-9.

4. Honjo S, Ajani JA, Scott AW, Chen Q, Skinner HD, Stroehlein J, et al. Metformin sensitizes chemotherapy by targeting cancer stem cells and the mTOR pathway in esophageal cancer. Int J Oncol. 2014;45(2):567-74.

5. Storozhuk Y, Hopmans SN, Sanli T, Barron C, Tsiani E, Cutz JC, et al. Metformin inhibits growth and enhances radiation response of non-small cell lung cancer (NSCLC) through ATM and AMPK. Br J Cancer. 2013;108(10):2021-32.

6. Skinner HD, McCurdy MR, Echeverria AE, Lin SH, Welsh JW, O'Reilly MS, et al. Metformin use and improved response to therapy in esophageal adenocarcinoma. Acta Oncol. 2013:52(5):1002-9.

7. Skinner HD, Sandulache VC, Ow TJ, Meyn RE, Yordy JS, Beadle BM, et al. TP53 disruptive mutations lead to head and neck cancer treatment failure through inhibition of radiation-induced senescence. Clin Cancer Res. 2012;18(1):290-300.

8. Sikka A, Kaur M, Agarwal C, Deep G, Agarwal R. Metformin suppresses growth of human head and neck squamous cell carcinoma via global inhibition of protein translation. Cell Cycle. 2012;11(7):1374-82.

9. He X, Esteva FJ, Ensor J, Hortobagyi GN, Lee MH, Yeung SC. Metformin and thiazolidinediones are associated with improved breast cancerspecific survival of diabetic women with HER2+ breast cancer. Ann Oncol. 2012:23(7):1771-80.

10. Mei ZB, Zhang ZJ, Liu CY, Liu Y, Cui A, Liang ZL, et al. Survival benefits of metformin for colorectal cancer patients with diabetes: a systematic review and meta-analysis. PLoS One. 2014;9(3):e91818.

11. Spratt DE, Zhang C, Zumsteg ZS, Pei X, Zhang Z, Zelefsky MJ. Metformin and prostate cancer: reduced development of castration-resistant disease and prostate cancer mortality. Eur Urol. 2013;63(4):709-16.

12. Chen G, Xu S, Renko K, Derwahl M. Metformin inhibits growth of thyroid carcinoma cells, suppresses self-renewal of derived cancer stem cells, and potentiates the effect of chemotherapeutic agents. J Clin Endocrinol Metab. 2012;97(4):E510-20

13. Lee MS, Hsu CC, Wahlqvist ML, Tsai HN, Chang YH, Huang YC. Type 2 diabetes increases and metformin reduces total, colorectal, liver and pancreatic cancer incidences in Taiwanese: a representative population prospective cohort study of 800,000 individuals. BMC Cancer. 2011;11:20.

14. Stevens RJ, Ali R, Bankhead CR, Bethel MA, Cairns BJ, Camisasca RP, et al. Cancer outcomes and all-cause mortality in adults allocated to metformin: systematic review and collaborative meta-analysis of randomised clinical trials. Diabetologia. 2012;55(10):2593-603.

15. Sandulache VC, Hamblin JS, Skinner HD, Kubik MW, Myers JN, Zevallos JP. Association between metformin use and improved survival in patients with laryngeal squamous cell carcinoma. Head Neck. 2014;36(7): 1039-43.

16. Yen YC, Lin C, Lin SW, Lin YS, Weng SF. Effect of metformin on the incidence of head and neck cancer in diabetics. Head Neck. 2015;37(9):1268-73.

17. Foreman A, Lee DJ, McMullen C, de Almeida J, Muhanna N, Gama RR, et al. Impact of type 2 diabetes mellitus on survival in head and neck squamous cell carcinoma. Otolaryngol Head Neck Surg. 2017:194599817726756.

18. Wong K, Huang SH, O'Sullivan B, Lockwood G, Dale D, Michaelson T, et al. Point-of-care outcome assessment in the cancer clinic: audit of data quality. Radiother Oncol. 2010;95(3):339-43

19. Edge SB, Byrd DR, Compton CC, Fritz AG, Greene FL, Trotti A. AJCC Cancer staging manual. 7th ed. New York, NY: Springer; 2010.

20. Evans JM, Donnelly LA, Emslie-Smith AM, Alessi DR, Morris AD. Metformin and reduced risk of cancer in diabetic patients. BMJ. 2005;330(7503):1304-5.

21. Liu B, Fan Z, Edgerton SM, Yang X, Lind SE, Thor AD. Potent anti-proliferative effects of metformin on trastuzumab-resistant breast cancer cells via inhibition of erbB2/IGF-1 receptor interactions. Cell Cycle. 2011;10(17):2959-66.

22. Rozengurt E, Sinnett-Smith J, Kisfalvi K. Crosstalk between insulin/insulin-like growth factor-1 receptors and $\mathrm{G}$ protein-coupled receptor signaling systems: a novel target for the antidiabetic drug metformin in pancreatic cancer. Clin Cancer Res. 2010;16(9):2505-11.

23. Malek M, Aghili R, Emami Z, Khamseh ME. Risk of cancer in diabetes: the effect of metformin. ISRN Endocrinol. 2013;2013:636927.

24. Vander Broek R, Mohan S, Eytan DF, Chen Z, Van Waes C. The PI3K/Akt/ mTOR axis in head and neck cancer: functions, aberrations, cross-talk, and therapies. Oral Dis. 2015:21(7):815-25.

25. Kalender A, Selvaraj A, Kim SY, Gulati P, Brule S, Viollet B, et al. Metformin, independent of AMPK, inhibits MTORC1 in a rag GTPase-dependent manner. Cell Metab. 2010;11(5):390-401

26. Vazquez-Martin A, Oliveras-Ferraros C, Cufi S, Del Barco S, Martin-Castillo B, Lopez-Bonet $E$, et al. The anti-diabetic drug metformin suppresses the metastasis-associated protein CD24 in MDA-MB-468 triple-negative breast cancer cells. Oncol Rep. 2011;25(1):135-40.

27. Han J, Fujisawa T, Husain SR, Puri RK Identification and characterization of cancer stem cells in human head and neck squamous cell carcinoma. BMC Cancer. 2014;14:173.

28. Rego DF, Elias ST, Amato AA, Canto GL, Guerra EN. Anti-tumor effects of metformin on head and neck carcinoma cell lines: a systematic review. Oncol Lett. 2017;13(2):554-66.

29. Franciosi M, Lucisano G, Lapice E, Strippoli GF, Pellegrini F, Nicolucci A Metformin therapy and risk of cancer in patients with type 2 diabetes: systematic review. PLoS One. 2013;8(8):e71583.

30. Noto H, Goto A, Tsujimoto T, Noda M. Cancer risk in diabetic patients treated with metformin: a systematic review and meta-analysis. PLoS One. 2012;7(3):e33411.

31. Yin M, Zhou J, Gorak EJ, Quddus F. Metformin is associated with survival benefit in cancer patients with concurrent type 2 diabetes: a systematic review and meta-analysis. Oncologist. 2013;18(12):1248-55.

32. Lega IC, Austin PC, Gruneir A, Goodwin PJ, Rochon PA, Lipscombe LL. Association between metformin therapy and mortality after breast cancer: a population-based study. Diabetes Care. 2013:36(10):3018-26.

33. Suissa S, Azoulay L. Metformin and cancer: mounting evidence against an association. Diabetes Care. 2014;37(7):1786-8.

34. Margel D, Urbach D, Lipscombe LL, Bell CM, Kulkarni G, Austin PC, et al. Association between metformin use and risk of prostate cancer and its grade. J Natl Cancer Inst. 2013;105(15):1123-31.

35. Shrank WH, Patrick AR, Brookhart MA. Healthy user and related biases in observational studies of preventive interventions: a primer for physicians. J Gen Intern Med. 2011;26(5):546-50

36. Spratt DE, Beadle BM, Zumsteg ZS, Rivera A, Skinner HD, Osborne JR, et al. The influence of diabetes mellitus and metformin on distant metastases in oropharyngeal Cancer: a multicenter study. Int J Radiat Oncol Biol Phys. 2016;94(3):523-31.

37. Vitale-Cross L, Molinolo AA, Martin D, Younis RH, Maruyama T, Patel V, et al. Metformin prevents the development of oral squamous cell carcinomas from carcinogen-induced premalignant lesions. Cancer Prev Res (Phila). 2012;5(4):562-73. 
38. Luo Q, Hu D, Hu S, Yan M, Sun Z, Chen F. In vitro and in vivo anti-tumor effect of metformin as a novel therapeutic agent in human oral squamous cell carcinoma. BMC Cancer. 2012;12:517.

39. Aldea M, Craciun L, Tomuleasa C, Berindan-Neagoe I, Kacso G, Florian IS, et al. Repositioning metformin in cancer: genetics, drug targets, and new ways of delivery. Tumour Biol. 2014;35(6):5101-10.

40. He L, Wondisford FE. Metformin action: concentrations matter. Cell Metab. 2015;21(2):159-62.

\section{Publisher's Note}

Springer Nature remains neutral with regard to jurisdictional claims in published maps and institutional affiliations.

Ready to submit your research? Choose BMC and benefit from:

- fast, convenient online submission

- thorough peer review by experienced researchers in your field

- rapid publication on acceptance

- support for research data, including large and complex data types

- gold Open Access which fosters wider collaboration and increased citations

- maximum visibility for your research: over $100 \mathrm{M}$ website views per year

At BMC, research is always in progress.

Learn more biomedcentral.com/submissions 